\title{
Kastler's views on Pauli's contribution
}

BERTOLLOTI is mistaken when he attributes the development of the concept of 'spin' to Pauli in his 1920 paper. This paper deals with the quite different problem of paramagnetism. 'Electron spin' was in fact discovered in $\mathbf{1 9 2 5}$ by Uhlenbeck and Goudsmit. Why did this concept become necessary to atomic physicists? The extensive study of fine structure of spectral lines and the related anomalous Zeeman effect obliged physicists to introduce a new quantum number to stand alongside the principal quantum number $n$, which determines the energy of an atomic state, and the azimuthal quantum number $k$, which gives the angular momentum of a state, both in units of Planck's constant $h$ and both being integers. (Today we use $l$ where $l=k-1$.) The third quantum number, which we represent by the letter $j$, sometimes takes a half integer value - this came as a surprise at the time-the vector representing the total angular momentum of the atom.

In a lecture given in Germany in 1965, when he was awarded the Planck medal, Samuel Goudsmit gave his personal version of the discovery of 'electron spin'. He cited a passage from a letter sent to him in March 1926 from Copenhagen by his friend and colleague $\mathrm{L}$. H. Thomas. In this letter Thomas writes: "I believe that you and Uhlenbeck had the good luck to see your spinning electron published and discussed before Pauli knew of it. More than a year ago Kronig had conceived the rotating electron and had developed his idea; Pauli was the first person to whom he showed this paper. Pauli told him that it was a ridiculous assumption, and so it happened that the first person to see Kronig's paper was also the last one." And Thomas concludes the story by stating: "All this shows that God's infallibility is not extended to whom had nominated himself to be his vicarian on earth." German physicists have since composed the rhyme: "Der Kronig hatt 'den Spin entdeckt, Hatt' Pauli ihn nicht abgeschreckt." (Kronig would have discovered spin if Pauli had not discouraged him.)

There is another reason why Goudsmit adopted a very critical attitude towards Pauli. In his Nobel lecture of 1945 Pauli gave an explanation of the hyperfine structure of spectral lines by postulating the existence of a nuclear spin whose magnetic moment interacts with the moment of the electron cloud, an idea which he had published in 1924. He claimed that this publication had influenced Goudsmit and Uhlenbeck in their claim of an electron spin. Goudsmit maintained that Pauli had never considered an internal moment of the nucleus. He had especially not foreseen it for the proton. For him the nuclear angular momentum, with its small associated magnetic moment, was due uniquely to the orbiting electrically charged particles inside the nucleus, namely the protons and, as was assumed at that time, the electrons. So the discovery of electron spin as an internal momentum owed nothing to Pauli.

Pauli, in his young years, had been a severe judge for the physicists of his generation who feared his sharp criticism, and as we have seen his influence on the development of physics was not always a positive one. I made his personal acquaintance some years before his untimely death, meeting him at the Physics Summer School of Les Houches and in Varenna. In those days he had become a friendly and indulgent person. His exclusion principle, on which are built Fermi-Dirac statistics and the explanation of the shell structure of the electrons in an atom, remains a great achievement in the development of physics.

and observed an oscillating Hanle effect (depolarization of the resonant light). In his Note e Memorie di E. Fermi ${ }^{18}$, quoted by Bertolotti, Rasetti describes how

Fermi pointed out that, since the mercury resonance line showed an anomalous Zeeman effect with a Landé factor of $3 / 2$, the mercury atom should more likely precess with a frequency $3 / 2$ times higher than the Larmor frequency. The choice between the two alternatives might be decided by investigating the behaviour of the polarization under magnetic fields, of the intensity of about one gauss and a frequency of a few megacycles per second in approximate resonance with the precession frequency of the atom.

It thus seems that in 1925 the idea of Brossel's double-resonance experiment had already occurred to Fermi, but the fields applied (1-2 G) were insufficient to produce any appreciable resonance.

Brossel and I arrived at this method independently after an exchange of letters about the failure of attempts to investigate an excited state of mercury by Bitter's method; Brossel was working in Bitter's laboratory at Massachusetts Institute of Technology (MIT) at the time (1949). We published the principle together ${ }^{19}$, after which Brossel went on to perform the corresponding experiment and published the result. In my 1950 paper ${ }^{20}$, I suggested altering the population of the magnetic $m$-states in the ground-state of the atoms by irradiating them with polarized light; if the polarization was linear, a change would be produced between states of large and small $m$ (alignment), while if the polarization was circular (left- or righthanded), concentration of the atoms in either the negative or positive $m$-state (polarization magnetization of the ensemble of atoms) would be favoured.

As a young student, I had the opportunity of visiting Germany in 1922, where I bought Sommerfeld's book. I read it eagerly, being especially interested in the section on the "Auswahlprinzip and Polarizationsregel" ("selection principle and polarization rule") in which the thesis of Sommerfeld's Polish research student A. Rubinowicz is summarized. Optical pumping is just an application of this principle and rule.

In 1951, Brossel returned to Paris after six years abroad, three with Tolansky in Manchester and three with Bitter at MIT. 